\title{
Apical Hypertrophy with Massive Myocardial Fibrosis: Comparison with Electrocardiographic Changes
}

\author{
Shinichi Fusimoto, Yumi Komoto*, Yasuhiro Sakaguchi*, Reiko Mizuno*, Kazuhiro Dohi* and Hiroshi Nakano
}

We report the case of a 48-year-old woman with apical hypertrophy with massive myocardial fibrosis. She was admitted to our hospital because of general malaise. Echocardiographic examination showed asymmetrical apical hypertrophy, and an electrocardiogram showed a giant negative $T$ wave on $V_{3}-V_{6}$. Right ventricular endomyocardial biopsy revealed massive myocardial fibrosis. Apical hypertrophy can lead to disorders that vary in severity, including rare massive myocardial fibrosis.

(Internal Medicine 37: 457-462, 1998)

Key words: electrocardiogram, endomyocardial biopsy, apical hypertrophy

\section{Introduction}

Apical hypertrophy, a subgroup of hypertrophic cardiomyopathies, is characterized by selective apical myocardial wall thickening and a giant negative $\mathrm{T}$ wave on the anterior chest lead of a standard 12 lead electrocardiogram. This disease, which was first reported in Japan by Sakamoto et al (1) and Yamaguchi et al (2), often appears in middle age and is complicated by mild hypertension. Cases of this disease can include a wide spectrum of pathological lesions, ranging from severe changes such as a marked disarray of myocardium and interstitial fibrosis to very slight changes similar to those often seen in essential hypertension, such as slight interstitial edema and minor myocardial fibrosis (3). In this report, we describe a case with massive myocardial fibrosis in apical hypertrophy.

\section{Case Report}

A 48-year-old woman was referred to our hospital because of general malaise and dyspnea. She had a past history of uterectomy for the treatment of myoma uteri. In 1987, she exhibited high blood pressure and had received anti-hypertensive drugs. In April 1990, when she underwent a hysterectomy because of myoma uteri, the preoperative examination revealed cardiomegaly on chest $\mathrm{X}$-ray, high voltage and a giant negative $\mathrm{T}$ wave in leads $\mathrm{V}_{4}, \mathrm{~V}_{5}$ on electrocardiogram, and apical hypertrophy on a transthoracic echocardiogram. She was followed in the outpatient clinic. In January 1996, she developed general malaise and dyspnea and a 12 lead electrocardiogram showed atrial fibrillation. She was then admitted to our hospital for further evaluation. Physical examination revealed blood pressure of $132 / 98 \mathrm{mmHg}$ and a heart rate of 56 beats/min. A systolic ejection murmur (Levine 1/6) was heard at the third costal space of the left sternal border. Peripheral edema was absent. A chest X-ray showed cardiomegaly (cardiothoracic ratio of 59.6\%). Laboratory findings on admission were as follows: red blood cell count $474 \times 10^{4} / \mu \mathrm{l}$, hemoglobin $15.2 \mathrm{~g} /$ $\mathrm{dl}$, hematocrit $42.5 \%$, white blood cell count $8,300 / \mu \mathrm{l}$, platelet count $19.3 \times 10^{4} / \mu \mathrm{l}$, asparate aminotransferase (AST) $17 \mathrm{IU} / \mathrm{l}$, lactate dehydrogenase (LDH) $324 \mathrm{IU} / l$, creatinine kinase (CK) $108 \mathrm{IU} / \mathrm{l}$. Autoimmune antibodies, such as antinuclear antibodies, anti-Scl-70 antibodies, and anti RNP antibodies, were negative. Anti-viral antibodies were not significantly increased. Serum catecholamines were within normal range. The electrocardiogram in 1996 in Fig. 1A shows high voltage in the left lateral chest leads and ST-segment elevation on $\mathrm{V}_{4}, \mathrm{~V}_{5}$. Fig. 1B shows the electrocardiogram in 1990. $\mathrm{R}$ wave amplitude and $\mathrm{T}$ wave negativity was severe. Electrocardiographic changes between 1990 and 1996 are shown in Fig. 2. A negative T wave in $\mathrm{V}_{3}-\mathrm{V}_{6}$ in 1990 decreased in 1995 , and virtually disappeared in 1996. The amplitude of the $\mathrm{R}$ wave in $\mathrm{V}_{3}-\mathrm{V}_{6}$ decreased in 1995, but in 1996 recovered to the same level as in 1992 (Fig. 3). An echocardiogram (Fig. 4) showed an end-diastolic dimension of $5.5 \mathrm{~cm}$, an end-systolic dimension of $3.2 \mathrm{~cm}$, and an extremely narrow internal diameter at the apex of the left ventricle. The left ventricular wall thickness was $1.8 \mathrm{~cm}$ at the base and $4.2 \mathrm{~cm}$ at the apex. Table 1 shows the serial changes of echocardiographic parameters. Tl-201 myocardial scinti-

From the Department of Clinico-Laboratory Diagnostics and *the First Department of Internal Medicine, Nara Medical University, Kashihara Received for publication August 6, 1997; Accepted for publication February 3, 1998

Reprint requests should be addressed to Dr. Shinichi Fujimoto, the Department of Clinico-laboratory Diagnostics, Nara Medical University, 840 Shijocho, Kashihara, Nara 634-8522 


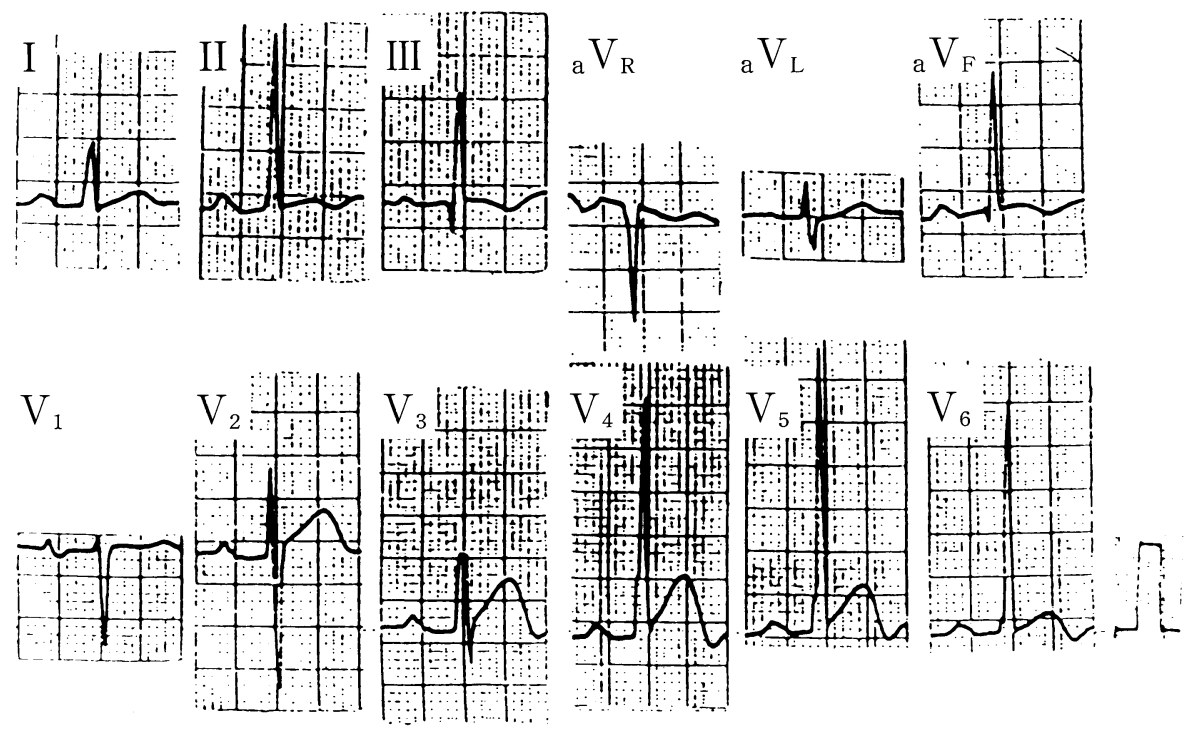

A
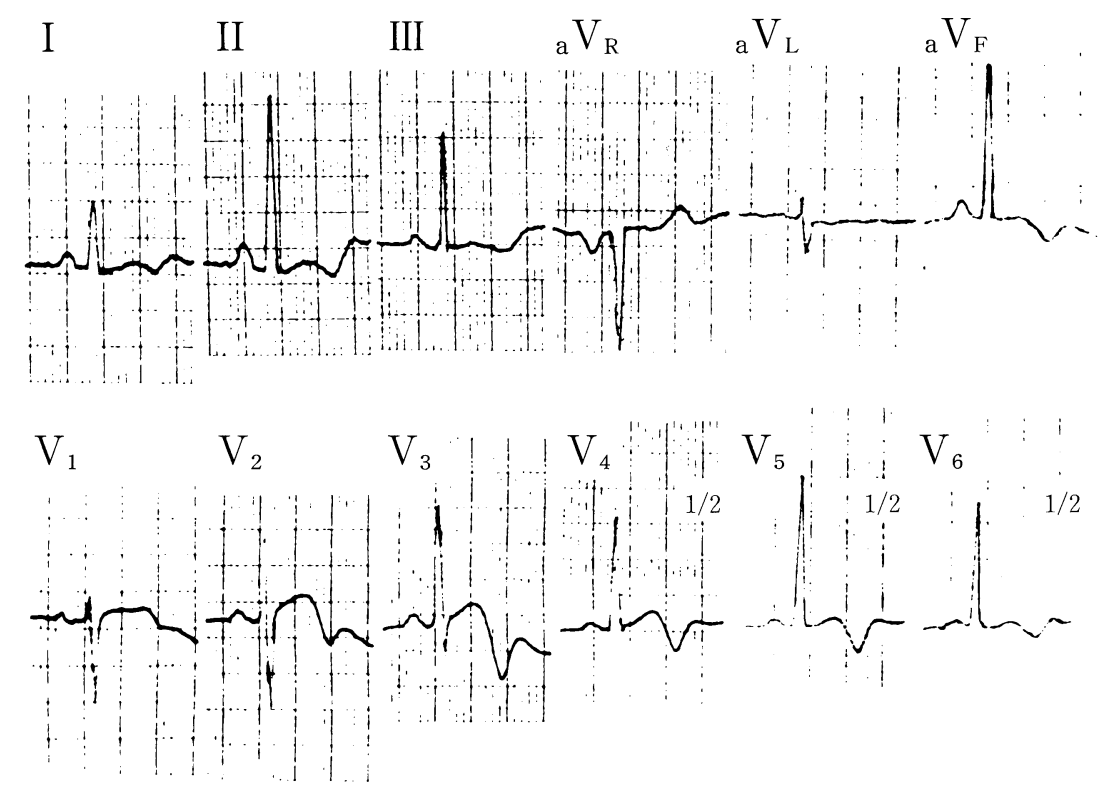

$\mathrm{B}$

Figure 1. Electrocardiograms. A) ECG in 1996, B) ECG in 1990, T wave negativity was decreased over a 6-year interval.

gram revealed an increased uptake in the apex, and the diffuse reduction of the washout rate (Fig. 5). Coronary angiogram showed no significant coronary artery stenosis. Left ventriculogram showed a spade-shaped appearance. A right ventricular endomyocardial biopsy specimen showed severe myocyte hypertrophy, disarrangement of the myocytes, and focal fibrosis in perimyocyte (Fig. 6).

\section{Discussion}

Apical hypertrophy, a disease that was recognized in Japan by Sakamoto et al (1) and Yamaguchi et al (2), is characterized by the apical hypertrophy on echocardiograms, and a giant negative $\mathrm{T}$ wave on a standard 12 lead electrocardiograms. Histological findings demonstrate myocardial lesions of varying severity $(3,4)$. Myocardial fibrosis is not a typical finding of this disease. The electrocardiographic changes in the present 
March 5th, 1992

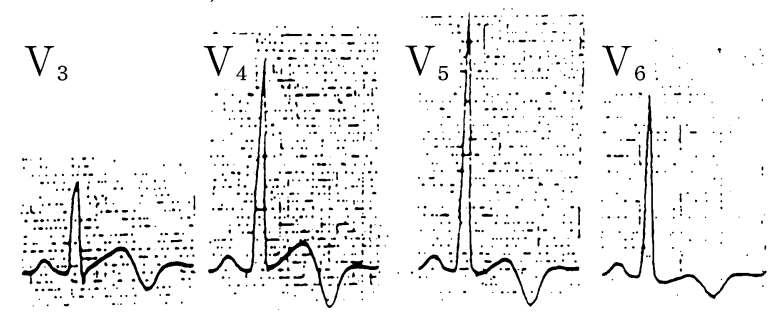

August 8th, 1995

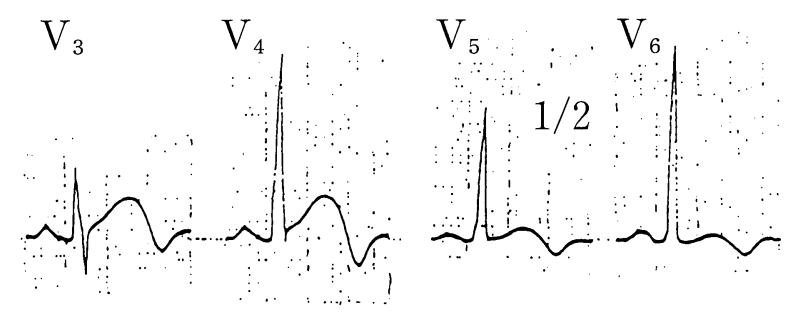

May 29th, 1996
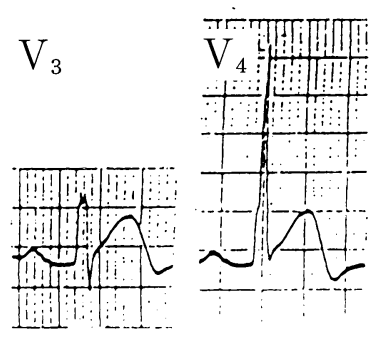

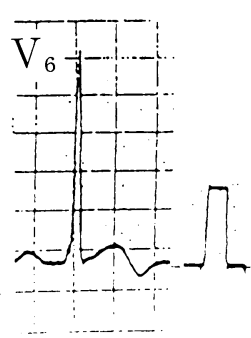

Figure 2. Changes in electrocardiogram, $V_{3}-V_{6}$ over a 19921996 period. A negative $T$ wave in $V_{3}-V_{6}$ in 1992 was decreased in 1995, and disappeared in 1996. The amplitude of the $R$ wave in $V_{3^{-}}$ $V_{6}$ decreased in 1995, but in 1996 recovered to the same level as in 1992.

case showed that the high voltage in a left lateral chest lead decreased transiently, and then increased, while the negative $T$ waves decreased consistently. The changes in voltage on the surface electrocardiogram may indicate the presence of myocardial fibrosis in this case.

\section{Electrocardiographic changes in apical hypertrophic car- diomyopathy}

Electrocardiographic changes in apical hypertrophy have been investigated in several clinical studies (5-7). Hasegawa et al showed that the left ventricular hypertrophy at the apex, especially with the posterior papillary muscle, is responsible for the negative $\mathrm{T}$ wave (8). On the other hand, the other reports indicate that the electrocardiographic changes are not uniform, and are not always related to myocardial hypertrophy. Nakamura et al (5) reported that increases in amplitude of the $\mathrm{R}$ wave and in negativity of the $\mathrm{T}$ wave in precordial leads reflect the

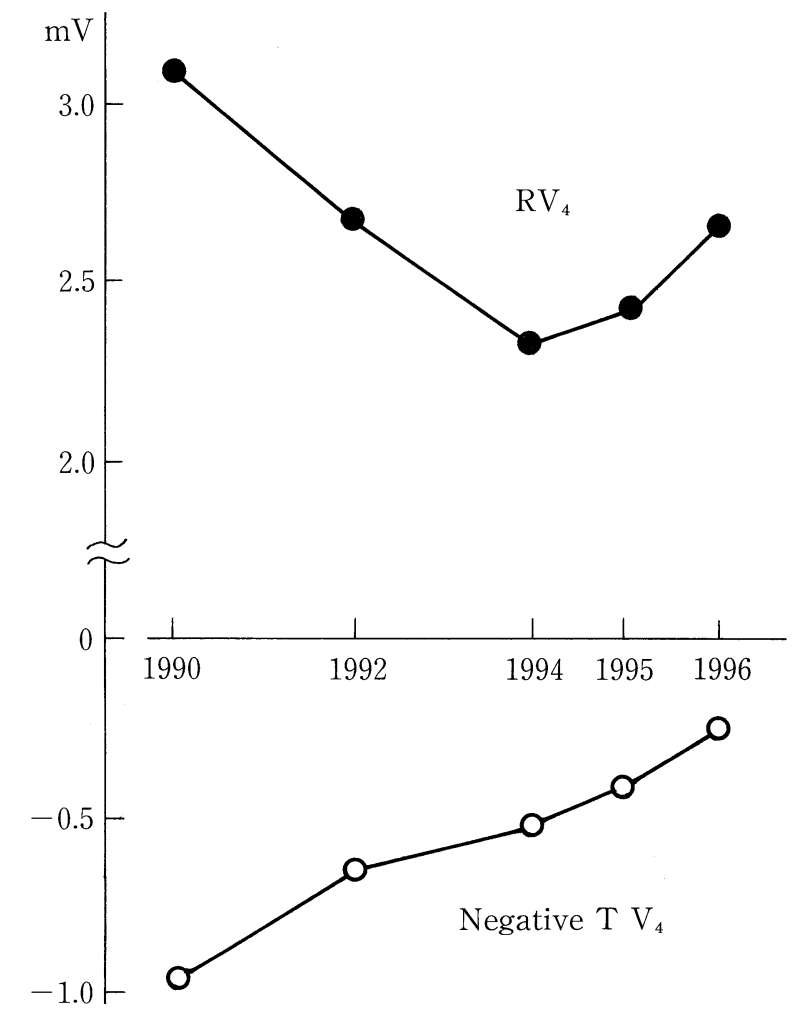

Figure 3. Changes of $R$ wave amplitude and $T$ wave negativity in $V_{4}$. $R$ wave in $V_{4}$ decreased once in 1994, and increased again in 1996. Negativity of $T$ wave in $V_{4}$ wave decreased from 1990 to 1996.

progression of left ventricular hypertrophy and conversely, a decrease in $\mathrm{R}$ wave amplitude shows degeneration of hypertrophied cardiac muscles. These electrocardiographic changes in apical hypertrophy, therefore, may signify a pathological process. In the present case, the negative $\mathrm{T}$ wave in $\mathrm{V}_{4}$ in 1992 disappeared in 1996; the amplitude of the $\mathrm{R}$ wave in $\mathrm{V}_{4}$ decreased once in 1995, and then increased again in 1996. ST segments were mildly elevated in $\mathrm{V}_{4}-\mathrm{V}_{6}$ in 1996 . We suggest that these changes imply the progression of myocardial fibrosis, with compensatory myocardial hypertrophy. The significance of the changes in T wave is still controversial in this type of hypertrophic cardiomyopathy. One report showed a day-to-day variation of the $\mathrm{T}$ wave, and found that $\mathrm{T}$ wave negativity is not always correlated with wall thickness in apical hypertrophy (9). Although there was no follow-up study of the pathological changes in apical hypertrophy in this case, a thallium myocardial scintigraphic study showed a reduction in perfusion in an apical lesion during a 2- to 5-year follow-up period (10). The present patient showed advanced myocardial fibrosis, which is not common in apical hypertrophic cardiomyopathy. Koga et al showed the results of the long-term follow-up of apical hypertrophy for more than 10 years, and showed the disappearance of giant negative $T$ waves to be associated with decreased $R$ wave amplitude(11). They speculate that these electrocardiographical 

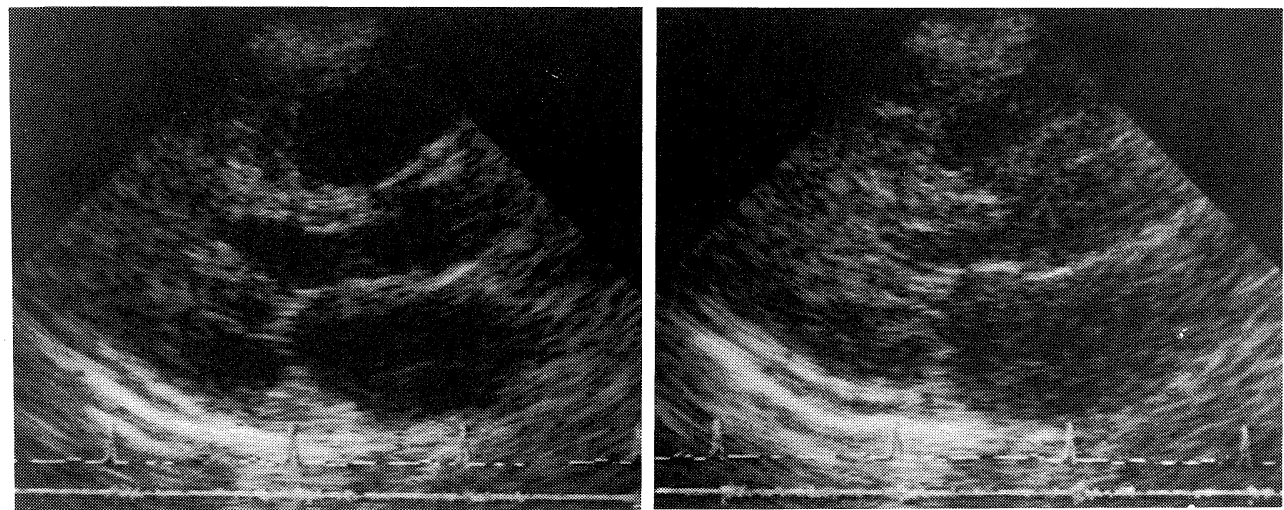

A
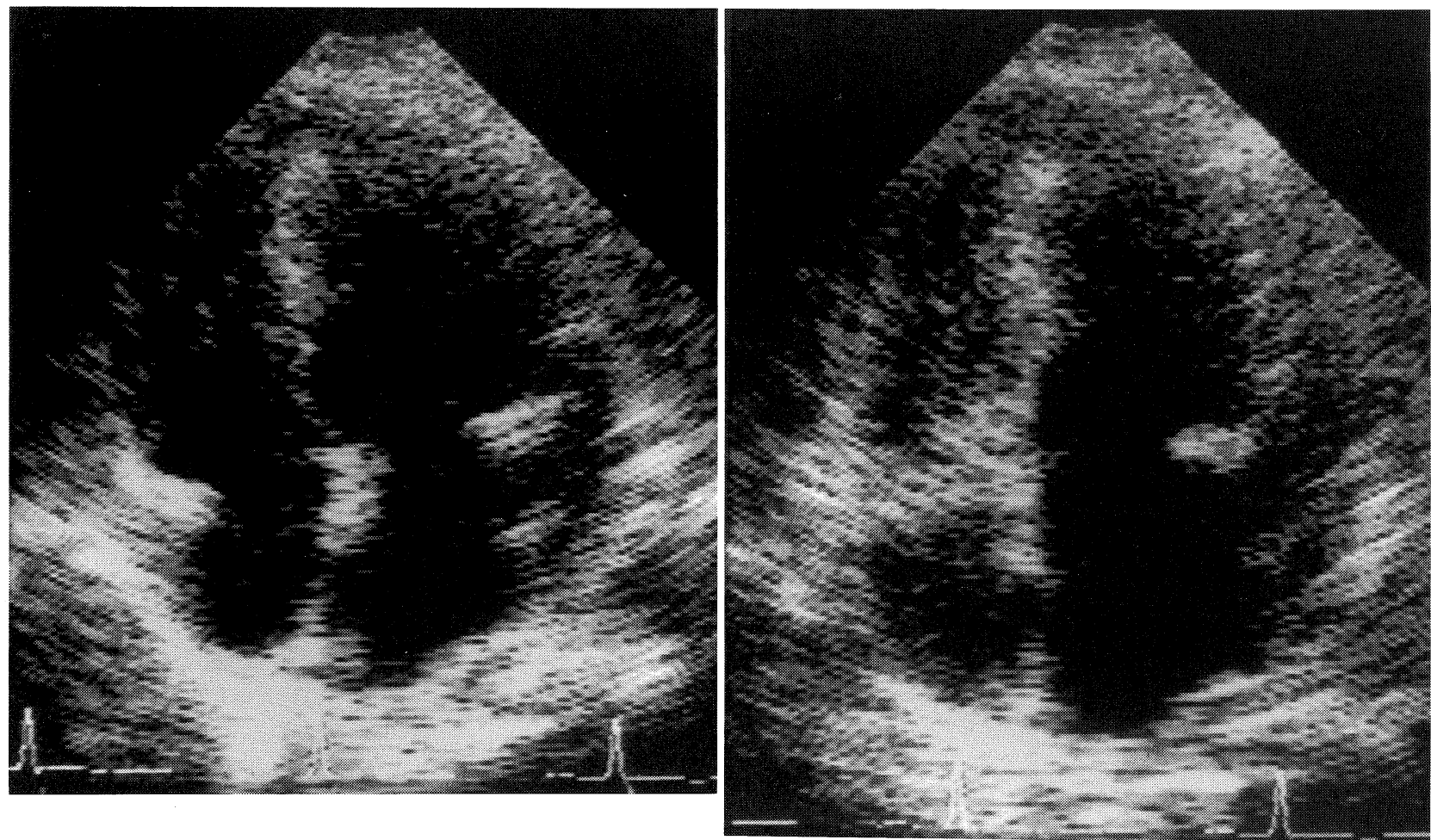

$\mathrm{B}$

Figure 4. Two-dimensional echocardiogram on admission. A) Left panel: parasternal long axis view at end-diastole, right panel: at end-systole. Internal diameter at the apex of the left ventricle was extremely narrow. B) Left panel: apical 4 chamber view at end-diastole, right panel: at end-systole. Spade shaped appearance of the left ventricle was observed.

changes depend on the progression of myocardial disease at the apex. We suspect that the mechanism of such electrocardiographic changes is due to myocardial fibrosis. Computer simulation study of electrocardiogram in the left ventricular hypertrophy showed that the amplitude of the $\mathrm{R}$ wave is related to an effective conduction rate, and the negative $T$ wave is related to the duration of action potential, and furthermore the conduction rate and duration of action potential can be independently related to the electrocardiographic changes (12). From this point of view, we suspect that the myocardial fibrosis could be represented on the electrocardiogram not only by the decreased amplitude of the $\mathrm{R}$ wave but also by the decreased negativity of the T wave. Electrocardiographic findings are potentially useful for detecting pathological changes in the myocardium noninvasively also in patients with apical hypertrophy.

\section{Myocardial fibrosis in apical hypertrophy}

A wide spectrum of pathological lesions in this type of 
Table 1. Changes of Echocardiographic Parameters

\begin{tabular}{lcccc}
\hline & 1993 & $' 94$ & $' 95$ & $' 96$ \\
\hline Dd (cm) & 4.0 & 4.2 & 4.9 & 5.5 \\
Ds (cm) & 2.2 & 2.2 & 2.9 & 3.2 \\
FS (\%) & 45 & 47 & 41 & 42 \\
\hline
\end{tabular}

Dd: left ventricular end-diastolic dimension, Ds: left ventricular end-systolic dimension, FS: fractional shortening.

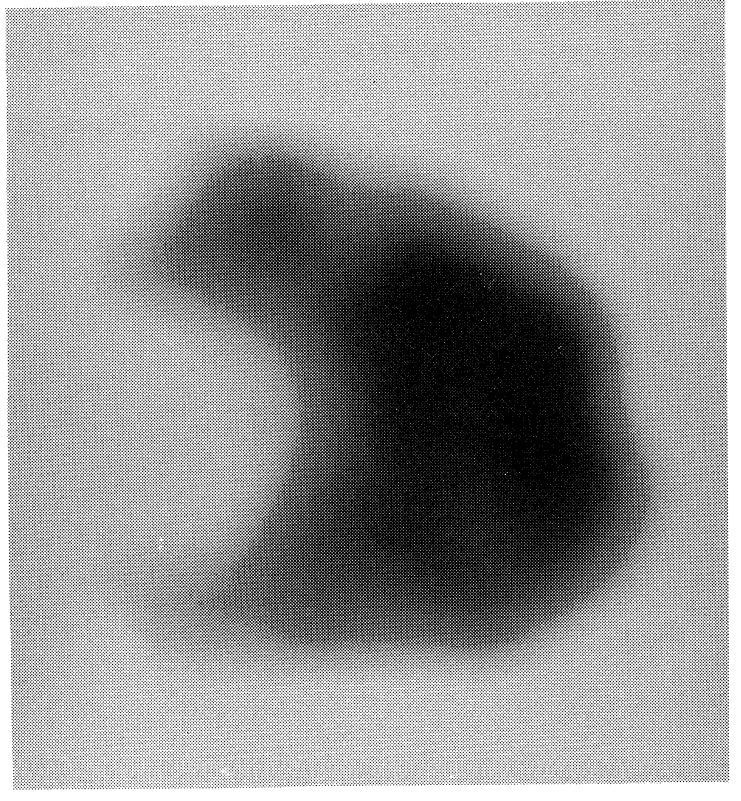

Figure 5. Tl-201 myocardial scintigram (lateral view). Increased uptake in the apex was obvious.

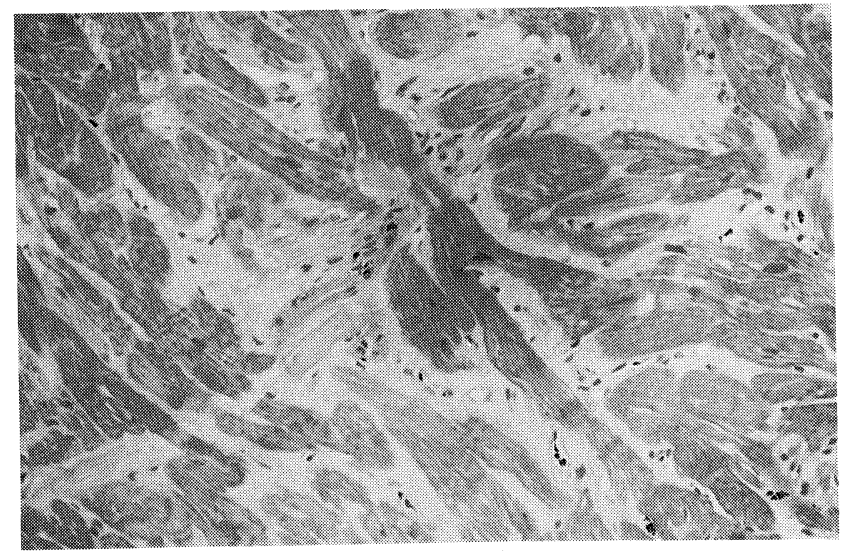

Figure 6. Light microscopic findings in biopsy of right ventricular endomyocardium. Biopsy specimen showed severe myocyte hypertrophy, disarrangement of the myocytes, and massive focal fibrosis in perimyocyte (HE stain, $\times 400)$. hypertrophic cardiomyopathy was reported by Nakanishi et al (3). Those authors found it difficult to make a diagnosis of apical hypertrophy based solely on pathological findings. In the present patient, interstitial fibrosis was severe, as compared with that in the typical case of apical hypertrophy. However, long-term follow-up of this disease has shown that some cases overlap progressive left ventricular dysfunction due to interstitial fibrosis, that results in severe heart failure. Myocardial fibrosis in hypertrophic cardiomyopathy is attributed to a decrease in compliance due to myocardial hypertrophy, elevated enddiastolic pressure, and subendocardial ischemia. The ischemia observed in patients with hypertrophic cardiomyopathy is thought to be due to spasm in the small coronary artery and to endomyocardial hypertrophy (13). Webb et al reported 26 patients with apical hypertrophic cardiomyopathy who were followed for a mean follow-up period of 7.3 years (14). They found that 21 of 26 patients were stable and only one patient with normal coronary arteries had an apical myocardial infarction; they suggested that a limited coronary flow reserve could be the mechanism for the ischemia in this disease. Hypertension is also considered to be a risk factor for myocardial fibrosis in apical hypertrophy (15). This patient also had a history of hypertension, but the disease was very mild, and would not explain the massive myocardial fibrosis observed. Although blood pressure during exercise was not very high and the serum catecholamines at rest were within the normal range in this case, the serum concentration of norepinephrine during exercise is considered to be elevated in apical hypertrophy, as compared with that in other forms of hypertrophic cardiomyopathy (16). The influence of catecholamines during exercise was thought to be not a major feature in the present case.

Cases of apical hypertrophy may progress to dilated cardiomyopathy $(17,18)$. Apical infarction may also develop possibly in apical hypertrophy (13). Taking the changes of echocardiographic parameters for six years into consideration, the present patient might be progressing from apical hypertrophy to dilated cardiomyopathy. Thus, in apical hypertrophy, we need to consider that massive myocardial fibrosis is also possible in the clinically stable condition.

\section{References}

1) Sakamoto $T$, Tei $C$, Murayama $M$, et al. Giant $T$ wave inversion as a manifestation of asymmetrical apical hypertrophy (AAH) of the left ventricle. Echocardiographic and ultrasono-cardiotomographic study. Jpn Heart J 17: 611, 1976.

2) Yamaguchi H, Ishimura T, Nishiyama S, et al. Hypertrophic non-obstructive cardiomyopathy with giant negative $\mathrm{T}$ waves (Apical hypertrophy): Ventriculographic and echocardiographic features in 30 patients. Am J Cardiol 44: 401, 1979.

3) Nakanishi S, Nishiyama S, Nishimura S, Yamaguchi H, Matsuya S. Histological features of apical hypertrophic cardiomyopathy. J Cardiol 15: 3, 1985 (in Japanese, Abstract in English).

4) Sumino $S$, Sugimoto $T$, Koide $T$, Murao $S$. Necropsy finding in a patient with hypertrophic cardiomyopathy. Jpn Heart J 26: 297, 1985.

5) Nakamura T, Furukawa K, Matsubara K, et al. Long-term follow-up of electrocardiographic changes in patients with symmetric apical hypertrophy. J Cardiol 20: 635, 1990 (in Japanese, Abstract in English).

6) Moro E, D'Angelo G, Nicolosi GL, Mimo R, Zanuttini D. Long term 
evaluation of patients with apical hypertrophic cardiomyopathy. Correlation between quantitative echocardiographic assessment of apical hypertrophy and clinical-electrocardiographic findings. Eur Heart J 16: 210 , 1995.

7) Sakamoto T, Amano K, Hada Y, et al. Asymmetrical apical hypertrophy: ten years experience. Postgrad Med J 62: 567, 1986.

8) Hasegawa K, Sawayama T, Nezuo S, Tadaoka S, Kawahara Y, Inoue S. Possible pathogenesis of giant negative $\mathrm{T}$ and negative $\mathrm{U}$ waves in hypertrophic cardiomyopathy: A report of two cases. J Cardiol 22: 271, 1992 (in Japanese, Abstract in English).

9) Fujii J, Saihara S, Sawada H, Aizawa T, Kato T. Distribution of left ventricular hypertrophy and electrocardiographic findings in patient with so-called apical hypertrophic cardiomyopathy. J Cardiol 15: 23, 1985 (in Japanese, Abstract in English).

10) Terashima $T$, Nakamura $T$, Furukawa $K$, et al. Regional myocardial coronary blood flow reserve in hypertrophic cardiomyopathy assessed by digital subtraction coronary angiography. J Cardiol 22: 113, 1992 (in Japanese, Abstract in English).

11) Koga $Y$, Katoh A, Matsuyama K, et al. Disappearance of giant negative $\mathrm{T}$ waves in patients with the Japanese form of apical hypertrophy. J Am Coll Cardiol 26: 1672, 1995.
12) Yamaki M, Nagata $Y$, Watanabe $S$, Tachibana $H$, Kubota I, Tomoike $H$. Mechanism of body surface electrocardiogram in the left ventricular hypertrophy: a study using three dimensional computer simulation model (abstract). Jpn J Electrocardiol 16: 572, 1996 (in Japanese).

13) Maron BJ, Epstein SE, Roberts WC. Hypertrophic cardiomyopathy and transmural myocardial infarction without significant atherosclerosis of the extramural coronary arteries. Am J Cardiol 43: 1086, 1979.

14) Webb JG, Sasson Z, Rakowski H, Liu P, Wigle D. Apical hypertrophy: clinical follow-up and diagnostic correlates. J Am Coll Cardiol 15: 83, 1990.

15) Fujiwara S, Umemoto M, Miyamoto $Y$, et al. The role of hypertension in apical hypertrophy. J Cardiol 15: 53, 1985 (in Japanese, Abstract in English).

16) Sugishita $Y$, Iida $K$, Matsuda $M$, et al. Apical hypertrophy and catecholamine. J Cardiol 15: 75, 1985 (in Japanese, Abstract in English).

17) Yutani $C$, Imakita $M$, Ishibashi-Ueda $H$, et al. Three autopsy cases of progression to left ventricular dilatation in patients with hypertrophic cardiomyopathy. Am Heart J 109: 545, 1985.

18) Beder SD, Gutgesell HP, Mullins CE, McNamara DG. Progression from hypertrophic obstructive cardiomyopathy to congestive cardiomyopathy. Am Heart J 104: 155, 1982. 